\title{
The effects of measurement errors in the restoring force feedback during real-time hybrid simulations
}

\author{
A. A. Sorkhabi \& O. Mercan \\ Department of Civil Engineering, University of Toronto, Canada
}

\begin{abstract}
Real-time hybrid simulation (RTHS) is a practical and economical experimental technique that integrates physical testing with computer simulation. In this method by dividing the structure into two parts, known as the experimental and analytical substructures, and synchronizing them, the equations of motion are solved in real-time. Thus, RTHS can capture the load-rate dependencies in an accurate manner. The implementation of RTHS involves challenges in accurate control of experimental substructure, execution of the testing algorithms in realtime as well as the synchronization of signals. One of these challenges is the measurement errors in restoring force feedback resulting from the random electrical noise that is usually inevitable in these testing platforms. Since the measured restoring force is used in command generation, RTHS suffers from error propagation affecting the accuracy and in some cases the stability of the simulation results. In this paper, using a recently developed user-reconfigurable computational/control platform at the University of Toronto, the effects of force feedback errors on the RTHS results will be investigated considering a wide range of experimental to analytical stiffness ratios. The accuracy of the RTHS results will be assessed using tracking indicators that reveal the phase and amplitude errors between the RTHS results and exact numerical solutions.

Keywords: real-time hybrid simulation, electrical noise, phase error, amplitude error, user reconfigurable computational/control platform.
\end{abstract}

\section{Introduction}

Experimental testing plays an influential role in dynamic performance evaluation of structures when they are subjected to extreme conditions such as earthquakes 
and explosions. When performed accurately the test results can facilitate the development of reliable analytical models; providing a valuable basis for predicting the behaviour of structural systems in similar situations. This, eventually, leads to the design and construction of safer and more reliable structures. Shake table, quasi-static, traditional pseudodynamic, and hybrid simulation are widely used experimental testing methods to assess the dynamic behaviour of structures. The last two methods combine physical testing with computer simulation.

Traditional pseudodynamic (PSD) test method was initially developed by Takanashi et al. [1] in the early 1970s and since then has been utilized and advanced by several researchers to conduct earthquake simulation studies [2-5]. In this method, a model of the test structure is constructed and tested physically while the mass and inherent damping of the system are modelled in a computer. The equations of motion are solved numerically using a step by step implicit or explicit time integration algorithms. The main advantage of implicit methods is that they are unconditionally stable however, for the cases that structure has nonlinear behaviour, the method needs to step forward with an iteration loop. In contrast, explicit methods are only conditionally stable. At each time step the resulting command displacements from the numerical solution are imposed to the test structure using displacement controlled hydraulic actuators. The corresponding restoring forces developed by the deformed structure are measured and fed back to the integration algorithm to be used in the next step command generation [2].

Hybrid simulation can be considered as a variation of traditional PSD. In this method the user is able to isolate and physically test those components of the system for which a reliable numerical model has not been developed yet (experimental substructure), while the rest of the system is modelled analytically (analytical substructure) in a computer [6]. If the test structure contains load-rate dependent components, the hybrid simulation should be carried out in real-time [7-9] that requires efficient and robust computational resources along with wellsynchronized data communication platform [9]. Real-time hybrid simulation (RTHS) enables the researchers to assess the dynamic behaviour of the complex structures including passive and semi-active control devices such as base isolators, tuned liquid and mass dampers, and MR dampers, etc. [10]. In RTHS, as only the critical components of the test structure need to be built and tested physically and remaining parts are modelled analytically, a wide range of influential parameters and loading cases could be considered in a timely and cost-effective manner [11].

The implementation of RTHS involves challenges in accurate control of experimental substructure and synchronization of the command and measured signals during the test to guarantee the accuracy and stability of the real-time hybrid simulation $[12,13]$. In a real-time PSD or hybrid simulation the restoring force feedback to the integration algorithm may contain amplitude error and/or time delay which are due to control errors in tracking the command displacement within the specified time to accommodate a real-time test, dynamic behaviour of the servo-hydraulic system, and any latency caused by the digital controller in 
receiving and executing commands [14]. Several researcher in real-time testing area have noticed that time delay in the measured restoring force has detrimental effects on the outer loop dynamics. Wallace et al. [15] determined that when the time delay in the restoring force in the system exceeds a critical value, that system becomes unstable. Wu et al. [16] investigated the stability of an SDOF system in presence of actuator delay during a real-time test. Horiuchi et al. [8] studied the effects of actuator delay in a real-time test, by means of energy balance approach and showed that a delay by an actuator causes increase in energy content of the system. The increase in energy was shown to be the same as that caused by a negative damping, and the system becomes unstable when the negative damping exceeds the inherent damping.

Another challenge in the implementation of the RTHS is the phenomenon called random errors in restoring force feedback resulting from electrical noise that is usually inevitable in these testing systems. Since the restoring force feedback is used in command generation, RTHS suffers from error propagation influencing the accuracy and in some cases the stability of the test results. In this paper the effects of force feedback errors due to random electrical noise on the RTHS results will be investigated. For this purpose, using a recently developed user-reconfigurable computational/control platform at the University of Toronto, a series of RTHS of a three story nonlinear structure with one degree of experimental substructure considering a wide range of experimental to analytical stiffness ratios will be conducted,. To evaluate the accuracy of the RTHS results, Phase and Amplitude Error Indices (PAEI) developed by Hessabi and Mercan [17] will be used. Although, PAEI was initially introduced as a tool to assess the accuracy of RTHS results considering command and measured displacements of the experimental substructure, it could be used to assess the accuracy of any two data sets against each other where it reveals the amplitude and phase errors between the two.

\section{Real-time hybrid simulation experimental setup}

\subsection{Hardware}

An overview of the RTHS test setup that was used in this study is shown in fig.1. The mechanical part of the test setup includes a hydraulic service manifold (HSM), rated at 120 gpm continuous flow, that regulates the supply oil pressure at $3000 \mathrm{psi}$, a fatigue rated hydraulic actuator with stroke length of \pm 5 inch $( \pm 127$ $\mathrm{mm})$ and maximum force capacity of $\pm 7,500 \mathrm{lbf}(33 \mathrm{kN})$ driven by an electro servo-valve with flow capacity of $16.5 \mathrm{gpm}$. A built-in \pm 5 inch $( \pm 127 \mathrm{~mm}) \mathrm{AC}$ LVDT and a dynamic load cell with a capacity of $\pm 12,500 \mathrm{lbf}(50 \mathrm{kN})$ are used to obtain the displacement and force feedbacks from the experimental substructures. 


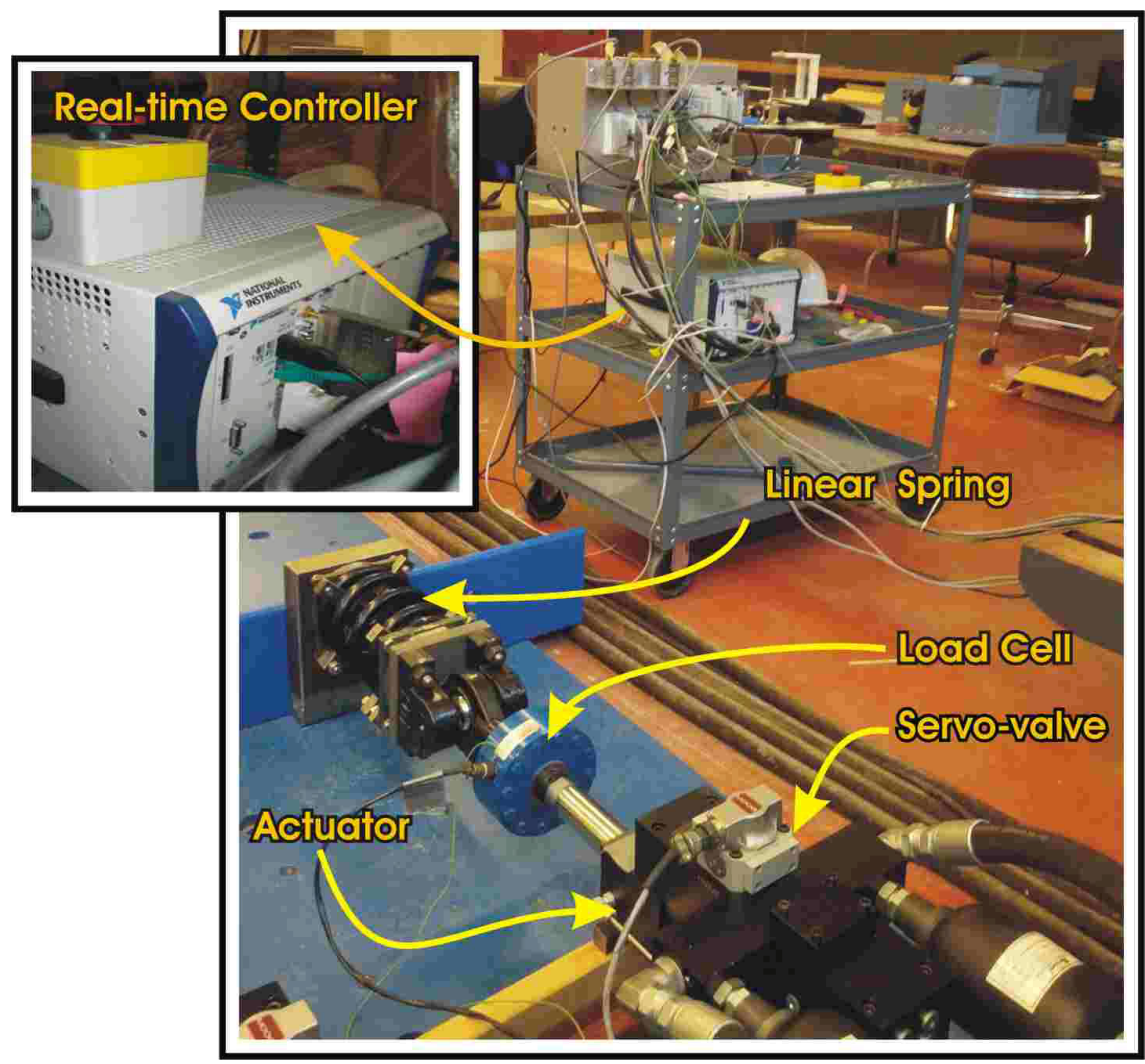

Figure 1: The components of the real-time testing system.

A quad-core real-time processor and a field programmable gate array (FPGA) are the key components that form the computational/control platform developed to implement the RTHS. The architecture of the designed controller and all associated signal routings are displayed in fig. 2 .

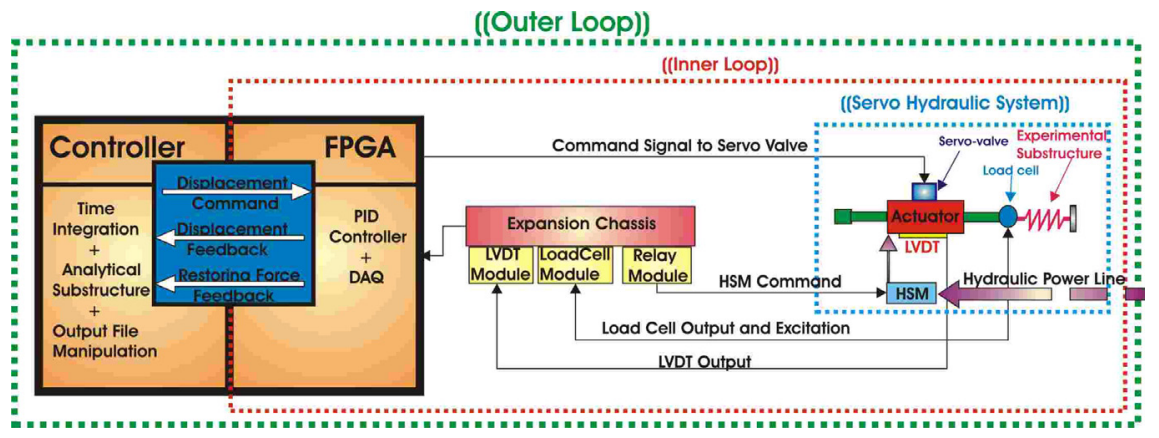

Figure 2: $\quad$ RTHS facility integrated control system architecture. 
As indicated in fig. 2 the process contains an inner loop within an outer loop. During a RTHS test, the command displacements to be imposed to the test structure for a given time step are determined in the outer loop by a numerical integration algorithm. The outer loop dynamics of the system is associated with the second order ordinary differential equation expressed by eqn (1).

$$
M \ddot{x}(t)+C \dot{x}(t)+R(x, \dot{x}, \ddot{x}, t)=F(t)
$$

where, $M$ is the mass matrix, $C$ is the damping matrix (representing the inherent structural damping), $R$ is the restoring force vector, $F$ is the effective or applied external force vector, and, are the velocity and acceleration vectors, respectively.

Also, the outer loop handles all the tasks related to analytical substructure and output file manipulations.

The inner loop is basically the servo-control loop of the system, in which the command displacements are imposed to the experimental substructure through the hydraulic actuator. All data communication with the hardware including the servo valve, LVDT and load cell is carried out in the inner loop [18].

\subsection{Software}

Unlike the turn-key controllers, the controller of the current setup has been selected as a flexible control/computational platform that must be configured to run specific tasks. Thus, along with the servo-control laws, several other tasks must be implemented to ensure safe start-up, satisfactory performance and safe shut-down of the system.

The software developed to conduct RTHS uses LabVIEW and MATLAB Simulink. The main part of the developed software resides on a multi-state Host VI (VI is the generic term used for codes developed in LabVIEW) called here as real-time VI, and an FPGA VI together with several sub VIs (equivalent to sub functions in MATLAB) all coordinated by a LabVIEW project [18]. Fig. 3 summarizes the tasks performed by the real-time and the FPGA VIs in the RTHS setup.

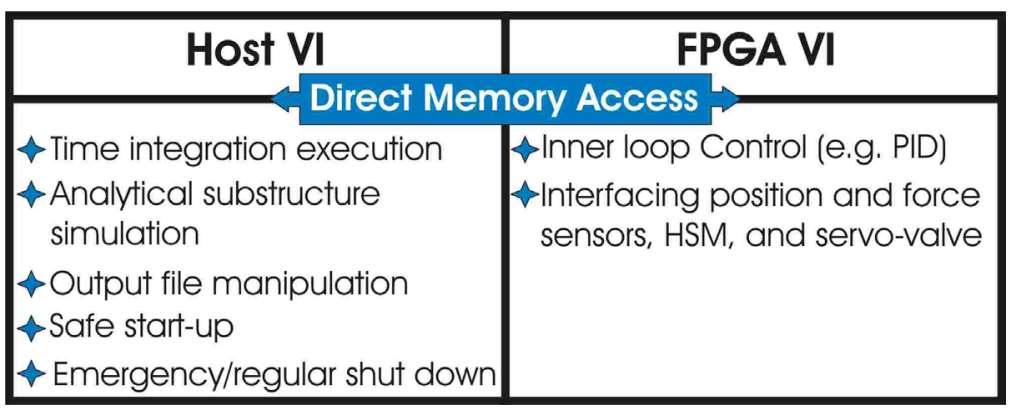

Figure 3: $\quad$ Summary of the tasks executed the host and the FPGA Vis.

The graphical user interface of the developed software enables the user not only to manipulate the influential control parameters, sensors' calibration 
constants, etc. and run the real-time hybrid simulation visually but also to control the hydraulics directly for safe start-up and emergency shut-down situations.

\section{Real-time hybrid simulation experiments}

\subsection{Test matrix}

Several real-time hybrid simulations were conducted to assess the accuracy of simulation results while there are random errors in restoring force - feedback due to electrical noise that is inevitably present. The test matrix is given in table 1. It includes twenty four tests considering a wide range of physical to analytical stiffness ratios in three different first-floor-drift levels.

\section{Table 1: $\quad$ Test matrix.}

\begin{tabular}{|c|c|c|c|c|c|c|c|c|}
\hline $\mathbf{K}_{\mathbf{e x p}} / \mathbf{K}_{\mathbf{1}}{ }_{\mathbf{~} \mathbf{f l o o r}}$ & $\mathbf{0 . 0 1}$ & $\mathbf{0 . 0 5}$ & $\mathbf{0 . 1}$ & $\mathbf{0 . 2}$ & $\mathbf{0 . 3}$ & $\mathbf{0 . 5}$ & $\mathbf{0 . 8}$ & $\mathbf{1 . 0}$ \\
\hline Maximum 1 $^{\text {st }}$ floor drift $=\mathbf{8 . 0} \mathbf{~ m m}, \boldsymbol{\delta}_{\mathbf{y}}=\mathbf{1 0 m m}$ & $\mathrm{T} .11$ & $\mathrm{~T} .12$ & $\mathrm{~T} .13$ & $\mathrm{~T} .14$ & $\mathrm{~T} .15$ & $\mathrm{~T} .16$ & $\mathrm{~T} .17$ & $\mathrm{~T} .18$ \\
\hline Maximum 1 $^{\text {st }}$ floor drift $=\mathbf{1 6 . 0} \mathbf{~ m m}, \boldsymbol{\delta}_{\mathbf{y}}=\mathbf{1 0 m m}$ & $\mathrm{T} .21$ & $\mathrm{~T} .22$ & $\mathrm{~T} .23$ & $\mathrm{~T} .24$ & $\mathrm{~T} .25$ & $\mathrm{~T} .26$ & $\mathrm{~T} .27$ & $\mathrm{~T} .28$ \\
\hline Maximum 1 $^{\text {st }}$ floor drift $=\mathbf{2 4 . 0} \mathbf{~ m m}, \boldsymbol{\delta}_{\mathbf{y}}=\mathbf{1 8 m m}$ & $\mathrm{T} .31$ & $\mathrm{~T} .32$ & $\mathrm{~T} .33$ & $\mathrm{~T} .34$ & $\mathrm{~T} .35$ & $\mathrm{~T} .36$ & $\mathrm{~T} .37$ & $\mathrm{~T} .38$ \\
\hline
\end{tabular}

A three story nonlinear moment resisting frame with an additional linear element in the first floor was considered as the test structure. In each hybrid test case, the three story nonlinear MRF was idealized as a mass-spring-dashpot hybrid tests, the experimental spring was identified using MTS loading machine in both tension and compression and it has a stiffness coefficient of $210 \mathrm{~N} / \mathrm{mm}$. In addition, the structural properties (e.g. mass, stiffness) of the analytical system and analytically modelled (analytical substructure) and a linear spring was physically tested (experimental substructure) that represented the linear element in the first floor of the test structure, as displayed in fig. 4. Prior to the substructure were configured such that the desired stiffness ratios in each test
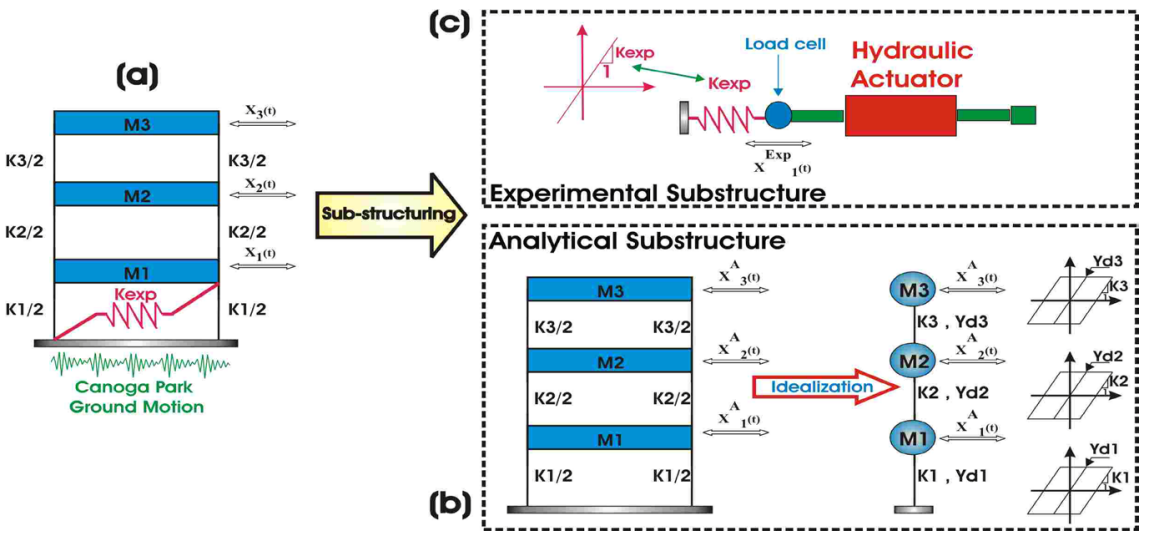

Figure 4: Real-time hybrid test, (a) structural substructure (c) experimental substructure. 
case could be achieved. The damping matrix of the analytical part was obtained assuming Rayleigh proportional damping with $2 \%$ damping ratio in the 1 st and 3rd modes. The floor yielding drift limit for the tests in first two rows (T.11 thorough T.28) of table 1 was set to $10 \mathrm{~mm}$ and for the third row test cases was set to $18 \mathrm{~mm}$.

An unconditionally stable implicit time-integration scheme based on the $\alpha$ method by Hilber et al. [19] that is implemented into real-time PSD framework through a fixed number of iterations [20] was programmed in the real-time VI.

The N196E component of the 1994 Northridge earthquake ground motion recorded at Canoga Park was used as the seismic input. It was scaled by appropriate scale factors to obtain the desired maximum story drifts listed in table 1. This way through results obtained from this study, both linear and nonlinear behaviour of the analytical substructure are represented.

\subsection{Results and discussion}

As an example, the RTHS results for test case T.23 are shown in fig. 5. In this case the structural properties of the analytical substructure were set such that the

Table 2: $\quad$ Structural and modal properties of analytical substructure.

\begin{tabular}{|c|c|c|c|c|c|}
\hline Floor & $\begin{array}{c}\text { Story Stiffness, K } \\
(\mathbf{N} / \mathbf{m})\end{array}$ & $\begin{array}{c}\text { Floor Mass, } \mathbf{M} \\
(\mathbf{k g})\end{array}$ & Mode & $\begin{array}{c}\text { Period } \\
\text { (sec) }\end{array}$ & Damping Ratio \\
\hline $\mathbf{1}$ & $2,100,000$ & $24,196.4$ & $\mathbf{1}$ & 1.31 & 0.02 \\
\hline $\mathbf{2}$ & $2,100,000$ & $24,196.4$ & $\mathbf{2}$ & 0.49 & 0.018 \\
\hline $\mathbf{3}$ & $1,750,000$ & $12,098.2$ & $\mathbf{3}$ & 0.37 & 0.02 \\
\hline
\end{tabular}
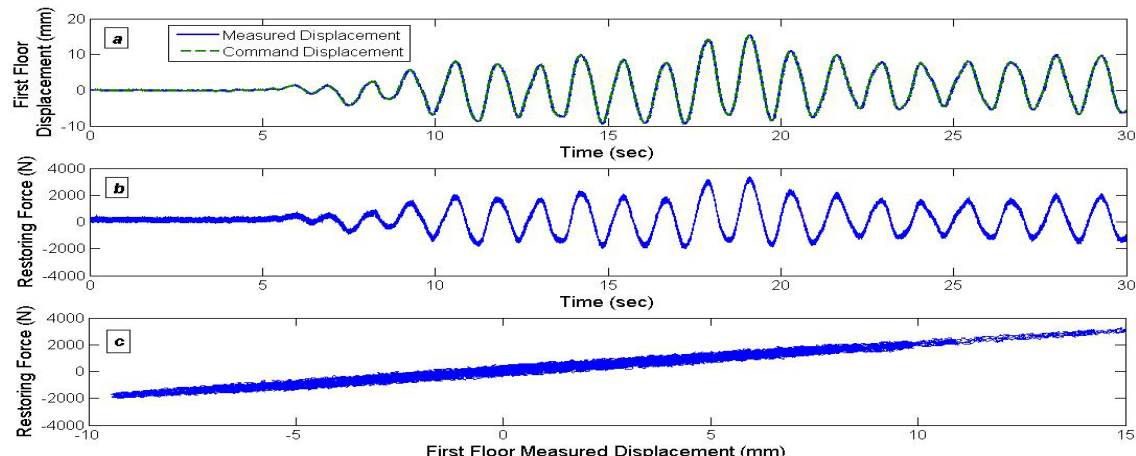

First Floor Measured Displacement (mm)

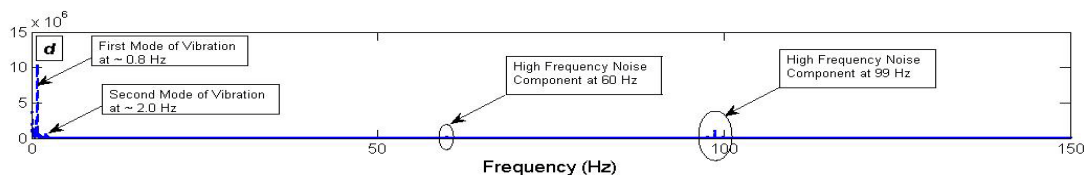

Figure 5: T.23 RTHS results, (a) $1^{\text {st }}$ floor command and measured displacement history, (b) restoring force history, (c) restoring force vs. measured displacement, (d) FFT of the restoring force. 
stiffness ratio between the experimental substructure and the first floor of the analytical substructure became 0.1 . The structural and modal properties of the analytical substructure are summarized in table 2 .

In fig. 5(a), first floor command and measured displacements are compared to each other over the simulation time. There is an excellent tracking of the command displacement performed by the servo control loop. The restoring force time history and its FFT are plotted in fig. 5(b) and fig 5(d), respectively. There is high frequency components $(60 \mathrm{~Hz}$ and $99 \mathrm{~Hz})$ embedded in the restoring force feedback that is basically due to the electrical power line. Considering the slope of the restoring force against the measured displacement plot in fig. 5(c), verifies the stiffness coefficient determined previously while identifying the spring by the loading machine.
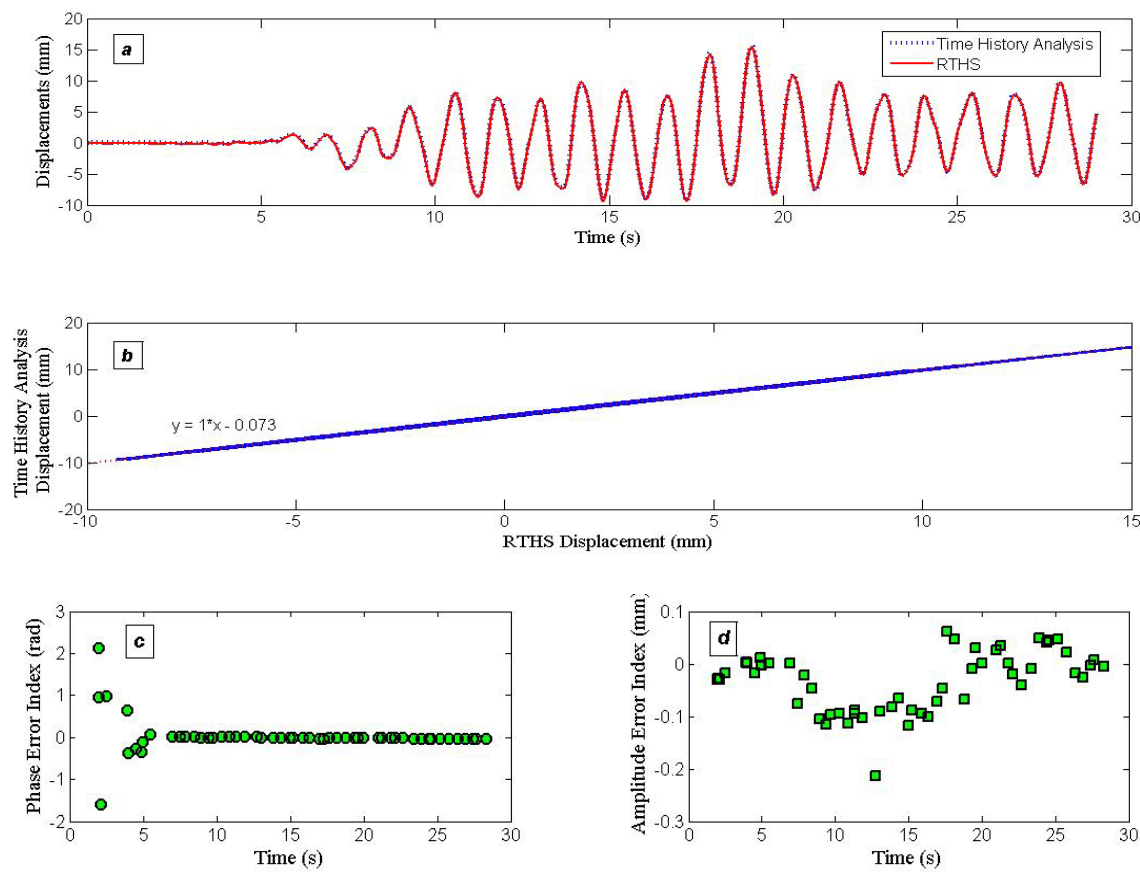

Figure 6: Comparison between RTHS results and time history analysis, (a) $1^{\text {st }}$ floor displacement history, (b) $1^{\text {st }}$ floor theoretical displacement vs. experimental displacement, (c) phase error index, (d) amplitude error index.

As the experimental substructure remained linear during the RTHS, it was possible to get a true answer through numerical simulation; here it is referred to as time history analysis. In fig. 6(a), first floor displacement history obtained from RTHS is compared to the time history analysis. It can be seen that there is a very good agreement between experiment and numerical simulation. Taking the advantage of synchronization subspace plot (SSP) concept developed by Wallace 
et al. [15] the force developed by the linear element in the time history analysis is plotted against the restoring force feedback from the experiment. In fig. 6(b) the SSP forms a slim hysteresis with an inclination of almost $45^{\circ}$ and a small enclosed area. This lies in the fact that there is a negligible amount of amplitude error and phase shift between RTHS and theoretical results. Furthermore, both amplitude error and phase shift between the experimental and theoretical signals are quantified using amplitude and phase error indices, as shown in fig. 6(c) and fig. 6(d) which agree with the observations obtained from fig. 6(b). The RMS values of the amplitude and phase errors for this test case are $0.072 \mathrm{~mm}$ and 0.022 radians, respectively.

The results from test T.23, where the experimental to analytical substructure stiffness ratio was only $10 \%$, reflect an acceptable accuracy in terms of amplitude and phase tracking. However the detrimental effects of the random errors due to electrical noise appear more as the stiffness ratio increases and the experimental substructure contributes more in the overall structural response. The results of the entire test matrix are summarized in fig. 7. This implies that in the RTHS applications that the experimental substructure has a comparable stiffness to the analytical part, careful attention should be paid to the measurement errors in the load cell readings.
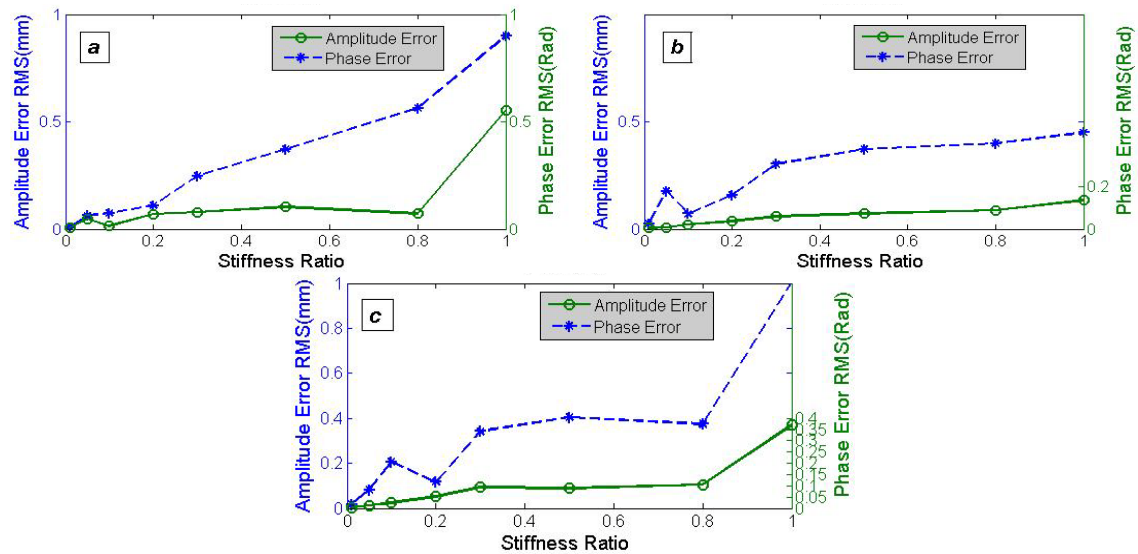

Figure 7: Amplitude and phase error RMS values vs. stiffness ratio for Max floor drifts of (a) $8 \mathrm{~mm}\{\mathrm{~T} .11$ through T.18\}, (b) $16 \mathrm{~mm}\{\mathrm{~T} .21$ through T.28\}, (c) $24 \mathrm{~mm}\{\mathrm{~T} .31$ through T.38\}.

It should be noted that in RTHS applications the restoring force errors are coupled with control errors (also known as tracking errors). However, due to the fact that the controller was well tuned prior to the testing and all test cases were conducted using identical control parameters, comparisons made between command and measured displacements for different cases showed an almost constant and negligible amount of tracking error. Therefore, the variation of error indices as shown in fig. 7 can be considered as a true representation of changes made in RTHS accuracy due to restoring force errors. In addition, as the random 
errors in the restoring force feedback were resulted from the electrical noise in the AC power line, their amplitude and frequency remained constant for all tests conducted.

\section{Conclusion and future work}

This paper presents the study done to investigate the effects of restoring force measurement errors on the RTHS results using a recently developed userreconfigurable computational/control platform. A comprehensive test matrix was selected considering a wide range of experimental to analytical stiffness ratios with the different maximum drift levels. In comparison to the time history analysis results, it was concluded that electrical noise introduces random errors in the restoring force feedback and has the potential to distort the RTHS results. Post processing the simulation results of all test cases using phase and amplitude error indicators revealed that as the experimental to analytical stiffness ratio increases, more detrimental effects are introduced by random errors in the RTHS results. Therefore special attention needs to be paid to minimize the measurement errors in the feedback signals.

\section{Acknowledgements}

The financial support for this study from NSERC Discovery (Grant 3716272009) and NSERC RTI (Grant 374707-2009 EQPEQ) programs, as well as the start-up funds from the University of Toronto is gratefully acknowledged. Any opinions, findings, conclusions and recommendations expressed here are those of the authors and do not necessarily reflect the views of the sponsors.

\section{References}

[1] Takanashi, K., Udagawa, K., Seki, M., Okada, T. and Tanaka, H., Nonlinear earthquake response analysis of structures by a computer actuator online system, Bulletin of Earthquake Resistant Structure Reasearch Center, No. 8, Institute of Industrial Science, University of Tokyo, Tokyo, Japan, 1975.

[2] Mahin, S.A. and Shing, P.B., Pseudodynamic method for seismic testing. Journal of Structural Engineering (ASCE), 111, pp. 1482-1503, 1985.

[3] Nakashima, M., Kaminosono, T. and Ishida, M., Integration techniques for substructure pseudodynamic test. Proc. of the $4^{\text {th }}$ US National Conference on Earthquake Engineering, pp. 515-524, 1990.

[4] Shing, P.B., Nakashima, M. and Bursi, O., Application of pseudodynamic test method to structural research. Earthquake Spectra, 12(1), pp. 29-56, 1996.

[5] Pinto, A.V., Pegon, P., Magonette, G. and Tsionis, G., Pseudo-dynamic testing of bridges using non-linear substructuring. Earthquake Engineering and Structural Dynamics, 33, pp. 1125-1146, 2004. 
[6] Dermitzakis, S.N. and Mahin, S.A., Development of substructuring techniques for on-line computer controlled seismic performance testing. Report UBC/EERC-85/04, Earthquake Engineering Research Center, University of California, Berkeley, CA, 1985.

[7] Nakashima, M., Kato, H. and Takaoka, E., Development of real-time pseudodynamic testing. Earthquake Engineering and Structural Dynamics, 21, pp. 79-92, 1992.

[8] Horiuchi, T., Inoue, M., Konno, T. and Namita, Y., Real-time hybrid experimental system with actuator delay compensation and its application to a piping system with energy absorber. Earthquake Engineering and Structural Dynamics, 28, pp. 1121-1141, 1999.

[9] Mercan, O. and Ricles, J.M., Experimental studies on real-time pseudodynamic (PSD) and hybrid PSD testing of structures with elastomeric dampers. Journal of Structural Engineering (ASCE), 135(9), pp. 1124-1133, 2009.

[10] Christenson, R., Lin, Y.Z., Emmons, A. and Bass, B., Large-scale experimental verification of semiactive control through real-time hybrid simulation. Journal of Structural Engineering (ASCE), 134, pp. 522-534, 2008.

[11] Ashasi-Sorkhabi, A., Malekghasemi, H. and Mercan, O. Implementation and verification of real-time hybrid simulation (RTHS) using a shake table for research and education. Submitted to Journal of Vibration and Control, 2012

[12] Mercan, O., Ricles, J.M. and Sause, R., Implementation of real-time hybrid pseudodynamic test method for evaluating seismic hazard mitigation measures. Proc. of Structures Congress (ASCE), Long Beach, CA, USA, 2007.

[13] Mercan O. and Ricles J.M. 2008. Stability Analysis for Real-time Pseudodynamic and Hybrid Pseudodynamic Testing with Multiple Sources of Delay. Earthquake Engineering and Structural Dynamics 37, pp. 12691293.

[14] Mercan, O. and Ricles, J.M., Stability and accuracy analysis of outer loop dynamics in real-time pseudodynamic testing of SDOF systems. Earthquake Engineering and Structural Dynamics 36, pp. 1523-1543, 2007.

[15] Wallace, M.I., Sieber, J., Nield, S.A., Wagg, D.J. and Krauskopf, B., Stability analysis of real-time dynamic substructuring using delay differential equations. Earthquake Engineering and Structural Dynamics, 34, pp. 1817-1832, 2005.

[16] Wu, B, Xu, G., Wang, Q. and Williams, M.S., Operator-splitting method for real-time substructuring testing. Earthquake Engineering and Structural Dynamics, 35, pp. 293-314, 2006.

[17] Hessabi, R.M. and Mercan, O., Phase and amplitude error indices for error quantification in pseudodynamic testing, Earthquake Engineering and Structural Dynamics, DOI: 10.1002/eqe.1186, 2012. 
[18] Ashasi-Sorkhabi, A. and Mercan, O., Real-time hybrid simulation verification with a user programmable computational/control platform., Proc. of 3rd International Structural Specialty Conference (CSCE), Edmonton, Alberta, Canada, 2012.

[19] Hilber, H. M., Hughes, T.J.R. and Taylor, R.L., Improved numerical dissipation for time integration algorithms in structural dynamics., Earthquake Engineering and Structural Dynamics, 5, pp. 283-292, 1977.

[20] Shing, P.B., Spacone, E. and Stauffer, E., Conceptual design of a fast hybrid test system at the University of Colorado., Proc. of the $7^{\text {th }}$ US Conference on Earthquake Engineering, Boston, MA, USA, 2002. 